\title{
Efficiency Analysis of Organic Light-Emitting Diodes Based on Optical Simulations
}

\author{
Tobias D. Schmidt, Bert J. Scholz, Christian Mayr, and Wolfgang Brütting
}

(Invited Paper)

\begin{abstract}
Organic light-emitting diodes (OLEDs) are promising new large-area light sources on their way to commercialization. However, there is still much room for improvement in terms of device efficiency and long-term stability under electrical operation. In this article, we review the current issue of efficiency analysis based on optical simulations of state-of-the-art OLED stacks. In detail, we present a method to determine the radiative quantum efficiency of the emitter, figure out the crucial points for non-isotropic emitter orientation and discuss the application of the developed method to analyze degradation effects during electrical operation.
\end{abstract}

Index Terms-device efficiency, optical properties, nonisotropic emitter orientation, degradation, organic light-emitting diodes.

\section{INTRODUCTION}

$\mathbf{O}$ RGANIC light-emitting diodes (OLEDs) offer promising applications in both display technology as well as in general lighting. The advantages of OLEDs are numerous, as it is possible to fabricate low-cost, lightweight, thin large-area devices, that could even be produced on flexible substrates such as polymer or metal foils and could achieve a semitransparent character. The thickness of the organic layers inside the diode comprises only a few $100 \mathrm{~nm}$ and thus the overall device thickness is limited only by the substrate and the encapsulation materials. The different organic materials are normally sandwiched between one (semi-)transparent and one reflecting (metallic) electrode. Applying a voltage of a few Volts to the electrodes results in charge carrier injection into and radiative recombination inside the device and therewith leads to electroluminescence from the emission layer of an OLED.

In 1963 the first observation of electroluminescence of an organic crystal was presented by Pope et al.[1]. However, several thousand Volts were needed to detect a clear luminescence signal from the $5 \mathrm{~mm}$ thick anthracene crystal. It took more than 20 years after these results until Tang and van Slyke had demonstrated the first efficient, low voltage driven OLED based on thin films from small organic molecules in 1987[2]. In 1990 the first solution processed polymer based OLED was developed by Burroughes et al.[3] and therewith the starting signal for a new, rapidly growing field of research had been given.

In the following years a huge amount of results has been published in this field of research and the efficiencies of OLEDs

Institute of Physics, University of Augsburg, 86135 Augsburg, Germany Corresponding author e-mail: tobias.schmidt@physik.uni-augsburg.de Corresponding author e-mail: wolfgang.bruetting@physik.uni-augsburg.de

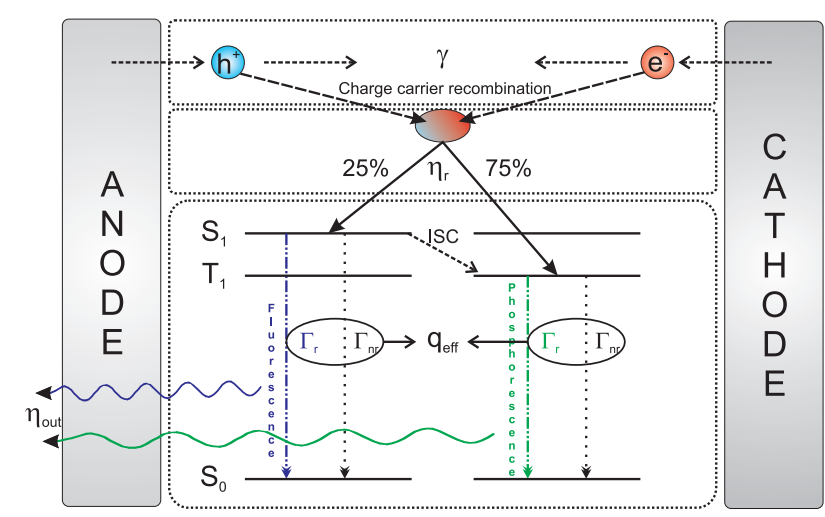

Fig. 1. Schematic illustration of the different factors determining the external quantum efficiency of organic light-emitting diodes. The acronyms are explained in the text.

could be increased continuously. Recently white OLED efficacies of $87 \mathrm{~lm} / \mathrm{W}$ could be demonstrated at brightnesses of $\approx 1000 \mathrm{~cd} / \mathrm{m}^{2}$ [4]. This value is close to that of fluorescent tubes and so the entry of OLEDs into the general lighting sector is predictable, since the small-area display market (e.g. for mobile phones) has already been flooded predominantly by AMOLED (Active Matrix OLED) displays[5].

Keeping in mind that about $1 / 6$ th of the total electrical power is consumed by lighting applications[6], it is crucial to develop energy-saving, long-lived lighting technologies such as OLEDs. Although the first commercial products for general lighting have been introduced by several companies[7] there is still much room for improvement in efficiency and lifetime. Furthermore, many physical effects, especially degradation processes, are not yet fully understood.

The scope of this article is to review optical simulations for achieving a comprehensive efficiency analysis of state-of-theart OLEDs. In particular we will discuss the importance of non-isotropic emitter orientations for reliable device analysis and highlight their potential for efficiency improvement. Furthermore, an innovative approach for degradation analysis will be presented.

\section{EXTERNAL QUANTUM EFFICIENCY OF OLEDS}

In terms of device physics the most important value of an OLED is its external quantum efficiency (EQE) that is given by the ratio between actually emitted photons from the device and the amount of injected charge carriers (see Fig. 1). The EQE of an OLED can be calculated using [8]

$$
\mathrm{EQE}=\gamma \cdot \eta_{\mathrm{r}} \cdot q_{\mathrm{eff}}(q) \cdot \eta_{\text {out }} .
$$


Therein $\gamma$ is the so-called charge carrier balance which is determined by the ratio between formed excitons and injected charge carriers. This factor can be close to unity if appropriate blocking layers are used in a multi-layer OLED.[9], [10] The second factor in this equation, $\eta_{\mathrm{r}}$, is the fraction of excitons that are quantum-mechanically allowed to decay under radiation of light with respect to spin selection rules. This factor is $1 / 4$ or 1 for fluorescent or phosphorescent emitters, respectively[11], [12]. Strictly speaking, $\eta_{\mathrm{r}}$ in fluorescent materials is not fixed and can be increased by several mechanisms, such as triplet-triplet-annihilation (TTA)[14], [15], [16] or thermally activated delayed fluorescence (TADF)[17], [18]. However, these phenomena are beyond the scope of this article and will not be discussed here.

The third factor, $q_{\text {eff }}$ is the effective radiative quantum efficiency that is determined by the intrinsic radiative quantum efficiency (RQE) - denoted as $q$ - of the emitting system in use and by the cavity effects in an OLED. The RQE of an emitting system is given by the radiative and the non-radiative decay rates of the emitting species, $\Gamma_{\mathrm{r}}$ and $\Gamma_{\mathrm{nr}}$, respectively:

$$
q=\frac{\Gamma_{\mathrm{r}}}{\Gamma_{\mathrm{r}}+\Gamma_{\mathrm{nr}}}
$$

and is only valid for an infinite bulk of the material and low excitation densities. Hence, if the emitting system is embedded in a microcavity, such as an OLED, an effective RQE must be considered:

$$
q_{\mathrm{eff}}=\frac{F \cdot \Gamma_{\mathrm{r}}}{F \cdot \Gamma_{\mathrm{r}}+\Gamma_{\mathrm{nr}}} .
$$

In this equation, $F$ represents the so-called Purcell factor that influences the radiative decay rate due to the Purcell effect and can be larger or smaller than unity. [19], [20], [21] Please note that the non-radiative processes are not influenced by the microcavity at all[22]. Therefore, the effective radiative quantum efficiency can be increased or decreased with respect to its intrinsic value inside an OLED, which should be taken into account in terms of efficiency analysis via optical simulations and, of course, for device improvement by optimization of the cavity length by proper modification of the layer thicknesses of the different materials. Furthermore, the effective RQE can be reduced drastically during electrical operation by quenching effects such as TTA or triplet-polaron-quenching (TPQ)[23]. Finally, $\eta_{\text {out }}$ describes the amount of photons that can leave the multi-layer system into the surrounding (air) medium. Due to the material and layered stack properties its value is typically in the range of $15 . .20 \%$ for planar, bottom emitting devices. The rest of the generated light is trapped inside the OLED due to total internal reflection and is lost either in substrate or waveguided modes[24] or dissipated to surface plasmon polaritons (SPPs) at a metal electrode[25], [26], [27].

In a more detailed analysis, other effects such as losses due to a reduced effective RQE (that can be far away from unity)[28] and changes of the power dissipation into the different optical channels due to orientation effects of the transition dipole moments of the emissive molecules[29], [30], [31] need to be considered. Especially the emitter orientation, a well-known feature in polymeric OLEDs[32], [33], was not taken into account for dye-doped small molecule OLEDs,

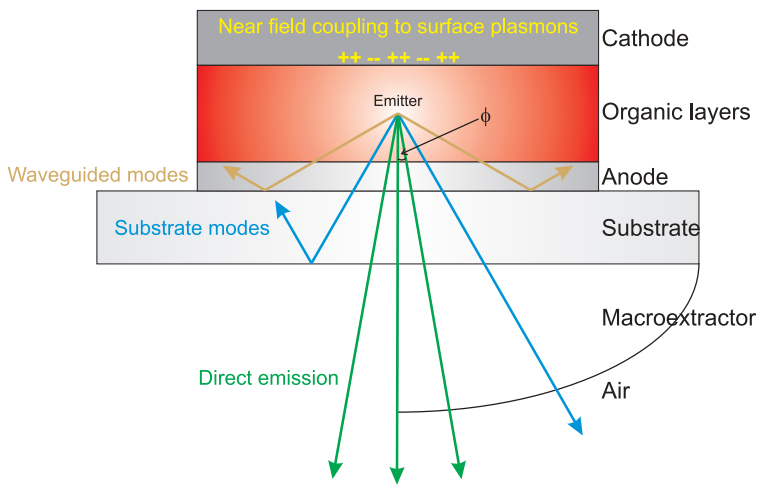

Fig. 2. Schematic illustration of the different optical modes in a simplified OLED structure. On the right half of the OLED a macroscopic index-matched outcoupling structure is attached to extract the substrate modes of the device. With this outcoupling enhancement a huge boost in the EQE can be achieved.

and especially those based on phosphorescent emitters, for a long time. However, recent studies have clearly shown that isotropic orientation of emitting molecules doped into molecular matrices is not generally the case[34]. E.g. the common red phosphorescent emitter Iridium(III)bis(2-methyldibenzo$[\mathrm{f}, \mathrm{h}]$ quinoxaline)(acetylacetonate) $\left(\operatorname{Ir}(\mathrm{MDQ})_{2}\right.$ (acac)) doped with a few wt \% into a N,N'-bis(naphthalen-1-yl)-N,N'bis(phenyl)benzidine ( $\alpha$-NPD) matrix shows a predominantly horizontal orientation of the emitting dipoles[35]. If this feature is not taken into account properly, the RQE of the emitting guest-host system is overestimated and hence the outcoupling factor of the OLED is underestimated. In consequence, predictions of the power dissipation into different optical modes by optical simulations will be erroneous, if an isotropic emitter orientation is assumed for devices comprising this emitting dye[36]. A detailed discussion of the power dissipation into different optical modes of an OLED will be given in the next section of this article.

\section{PRINCIPLES OF POWER DISSIPATION IN OLEDS}

\section{A. Optical modes in OLEDs}

Optical modeling and numerical simulations of the power dissipation between the optical modes of OLEDs have been a fast grown field in recent years and a number of relevant papers have been published focusing on this special topic[37], [38], [39], [40], [41], [42], [43], [44], [45], [46]. The presentation in this article is mainly based on the theoretical considerations of Ref. [47] and the main focus will lie on the implementation of non-isotropic emitter orientation and analyzing degradation effects.

Figure 2 shows the different optical modes of a simplified OLED stack to which an excited molecule can dissipate its energy. The first mode is the direct emission to air. The amount of power that is coupled to this channel is determined by total internal reflection at the substrate/air interface and therewith by the refractive index of the (glass) substrate and the angular dependent emission profile of the emissive molecules. Strictly speaking, the refractive index of the emissive layer can influence the outcoupling efficency, too, particlularly if a matrix material with a low refractive index is used. However, 


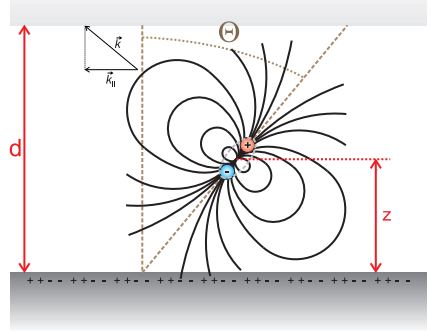

(b)

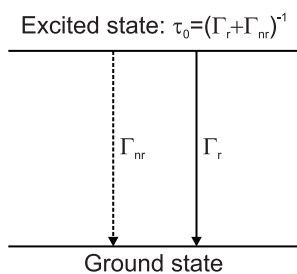

Fig. 3. (a) Sketch of an oscillating electrical dipole embedded in a (dielectric) layer, which is sandwiched between two interfaces. Near field-coupling to surface plasmons appears at the interface to the lower layer, due to its metallic character. Parameters that are influencing the emission from the radiating dipole are the cavity length $\mathrm{d}$, the distance $z$ of the dipole to the metal, the relative orientation $\Theta$ of the transition dipole moment to the surface normal, the wavevector $\vec{k}$ of the emitted electromagnetic wave, and its inplane component $\overrightarrow{k_{\|}}$. (b) Quantum mechanical analogue. The two-level system consists of an excited state of the molecule and the ground state with radiative and non-radiative decay rates between them, leading to a finite lifetime of the excited state. If the dipole is embedded in a cavity, the radiative rate will be modified by the Purcell factor $F: \Gamma_{\mathrm{r}}^{*}=F \cdot \Gamma_{\mathrm{r}}$. Hence, the excited state lifetime can be described by $\tau=\left(\Gamma_{\mathrm{r}}^{*}+\Gamma_{\mathrm{nr}}\right)^{-1}$.

in a typical OLED with an isotropic emitter orientation the internal light escape cone is about $30^{\circ}$ from the surface normal and less than $20 \%$ of the total power is radiated into this optical channel. Light that is emitted under higher angles is guided in substrate modes until it reaches the angle of total internal reflection due to the refractive index missmatch at the organic/substrate interface. For the sake of simplicity, we do not distinguish between the organic layers and the indium tin oxide (ITO) anode, that is typically used in bottom emitting OLEDs, in this discussion. However, this part of the dissipated power can be easily extracted by light outcoupling structures based on ray optics, such as glass hemispheres or outcoupling foils. The residual light, that is not coupled into one of the first two optical channels is captured inside the organic layers as waveguided modes or is absorbed by the organic layers. The remaining consumed (electrical) power is dissipated (non-radiatively) into vibronic modes of the molecules and/or the molecular lattice or is near-field coupled (radiatively) to surface plasmon polaritons at the organic/metal interface. The latter are one of the major optical loss channels in organic light-emitting diodes, especially if the emitting species is located close to metallic layers. Calculating the optical losses in a typical planar OLED stack with isotropic emitter orientation by integrating over the coupled power to waveguided modes and to SPPs results in values as high as $50 \%$ of the radiatively generated energy of the excited molecules[20], [25], [26].

\section{B. Optical modeling of light emission in OLEDs}

As mentioned before, one has to distinguish between an excited molecule in an infinite bulk material and in stratified media embedded in a microcavity structure, like an OLED. In the latter case, the emission of the molecules (fluorescence and/or phosphorescence) is modified by its optical surroundings by the so-called Purcell effect, that has been originally investigated in magnetic resonance experiments[48]. Later, the

importance of this effect for excited molecules near (reflecting) interfaces has been pointed out[49], [50]. The first theoretical principles of the situation of an excited molecule near a surface have been developed by Chance, Prock, and Silbey (CPS). In their approach the emitting molecules are treated as classical oscillating electrical dipoles near a dielectric interface[51], [52]. This is possible, because the radiated power of a classical dipole antenna corresponds to the quantum mechanical probability of the radiative decay under light emission of an excited molecule. This theoretical model was later expanded for ensembles of molecules embedded in multiple layer stacks with different optical constants and near metallic interfaces[27], [53], [37].

The optical simulation used in this study follows the formalism of Barnes and Wasey, which solves the 3-dimensional Maxwell equations for an excited dipole emitter ensemble surrounded by multiple thin and planar layers with respect to electromagnetic boundary conditions[54]. Physical input parameters are the layer thicknesses $\left(d_{i}\right.$, where $\sum d_{i}$ is the cavity length of the OLED), the complex refractive indices $\left(\mathrm{n}_{i}\right.$ and $\kappa_{i}$ ), the position of the excited molecule with respect to the different interfaces (especially the emitter/metal distance $z$ ), the emitter orientation $\Theta$ and the intrinsic radiative quantum efficiency $(q)$ of the emitting (guest/host) system (see Fig. 3). Using a transfer-matrix-formalism and taking into account the Fresnel reflection and transmission coefficients at the involved interfaces allows to calculate the power dissipation spectrum for a given dipole esemble.

If the emitting system is embedded in an OLED stack, the prescence of a microcavity changes two important emission parameters of the ensemble of emitter molecules. First, the radiative decay rate is changed by the Purcell factor $F$, resulting in an effective $\mathrm{RQE}$ of the emitting system as mentioned before (see eq. 3 ). This is equivalent to a changed excited states lifetime of the molecules. In accordance to Fig. $3 \mathrm{~b}$ ) the intrinsic excited states lifetime (if no interfaces and only low excitation densities are present) is defined as $\tau_{0}=\left(\Gamma_{\mathrm{r}}+\Gamma_{\mathrm{nr}}\right)^{-1} \equiv \Gamma_{0}^{-1}$, with $\Gamma_{\mathrm{r}}$ representing the radiative and $\Gamma_{\mathrm{r}}$ the non-radiative decay rate of the emitting molecules. If the emitting system is embedded into a microcavity-like enviroment such as an OLED, the Purcell effect is changing the radiative rate and thus the modified excited states lifetime can be written as:

$$
\tau=\frac{1}{F \cdot \Gamma_{\mathrm{r}}+\Gamma_{\mathrm{nr}}} \equiv \Gamma^{-1}
$$

Second, the microcavity changes the power distribution between the different optical channels, such as direct emission and substrates modes, determining the outcoupling factor of the device $\eta_{\text {out }}$.

Following from these basic principles, the radiated power can be expressed as the relative decay rate of an isotropically oriented ensemble of non-interacting excited molecules emitting at one distinct wavelength inside a cavity $(\Gamma)$ as compared to in an infinite bulk $\left(\Gamma_{0}\right)$

$$
\tilde{P}(\lambda, z)=\frac{\Gamma}{\Gamma_{0}}=(1-q)+q \cdot \int_{0}^{\infty} P\left(k_{||}, \lambda, z\right) d k_{||}
$$




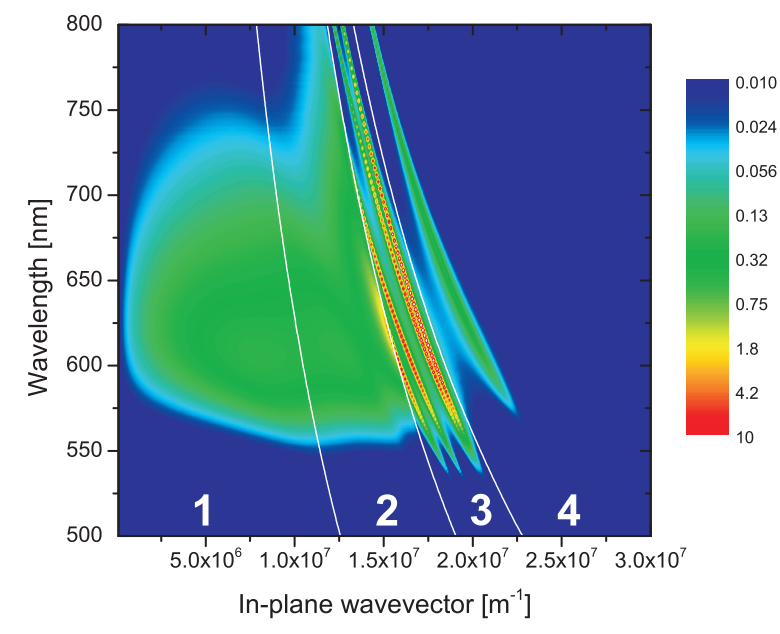

Fig. 4. Simulated power dissipation spectra of a red state-of-the-art OLED stack as a function of the emitted wavelength and the in-plane wavevector. Colored areas are indicating different intensities of the emission. The dashed white lines represent the boundaries between different optical modes of the device; 1: direct emission to air, 2: light, that is captured in the substate, 3: waveguided modes, and 4: coupled energy to surface plasmons polaritons at the cathode/ETL interface.

wherein $P\left(k_{\|}, \lambda, z\right)$ is the specific power dissipation function, which depends on the component of the wavevector that is projected onto the plane of the device surface $k_{\|}$, the emitted wavelength, and the emitter position in the OLED stack $z$ (see Fig. 3). Due to the very small thickness of the emission layer typically used in OLEDs based on small molecules $(\approx 10-20 \mathrm{~nm})$, we are using a $\delta$-shaped emission zone in all the simulations presented in this article. However, it is straightforward to implement spatial profiles such as Gaussian or exponential functions for the emission zone, which will be very important for polymeric OLEDs, just by simulating $\delta$ shaped emission profiles at different positions $(z)$ inside the OLED cavity and averaging these results with the pre-defined shape function[55].

Figure 4 shows the power dissipation spectra of a typical red state-of-the-art OLED stack. In this simulation a $\delta$-shaped emission zone was assumed, the RQE was set to unity and an isotropic orientation of the emissive dipole moments of the emitter was supposed. The power dissipation spectrum was first calculated for a "white" spectrum, for which the intensity at each wavelength has been set to the same value between $400 \mathrm{~nm}$ to $800 \mathrm{~nm}$ and was later on weighted with the normalized intrinsic emitter spectrum $S(\lambda)$.

In agreement with Eq. 5 one can now calculate the total emitted power of the emissive system by using the simulated power dissipation spectrum and integrating over all relevant wavelengths and in-plane wavevectors:

$$
\tilde{P}_{\text {tot }}(z)=(1-q)+q \cdot \int_{\lambda_{1}}^{\lambda_{2}} S(\lambda) \int_{0}^{\infty} P\left(k_{\|}, \lambda, z\right) d k_{\|} d \lambda .
$$

Note that $\tilde{P}_{\text {tot }}(z)$ obviously depends on the layer structure of the OLED, including all layer thicknesses and their optical constants as well as the emitter position. For sake of simplicity these parameters are summarized in a single variable $z$. With the definitions of the intrinsic and the modified total decay rates in an infinite medium and inside a cavity, respectively, it follows that the spectrally wheigted integral over the power dissipation directly yields the Purcell factor $F$. Thus Eq. 6 can be rewritten as

$$
\tilde{P}_{\text {tot }}=(1-q)+q \cdot F .
$$

Again note that just like $\tilde{P}_{\text {tot }}$, also the Purcell factor depends on the stack layout of the OLED, i.e. $F=F(z)$, though not written explicitly here and in the following. Therewith, the relative changes of the radiative quantum efficiency are given by:

$$
\frac{q_{\mathrm{eff}}}{q}=\frac{F \cdot\left(\Gamma_{\mathrm{r}}+\Gamma_{\mathrm{nr}}\right)}{F \cdot \Gamma_{\mathrm{r}}+\Gamma_{\mathrm{nr}}}=F \cdot \frac{\Gamma_{0}}{\Gamma}=\frac{F}{F \cdot q+(1-q)} .
$$

We note that in this terminology the coupling of the excited molecules to modes of the cavity that do a priori not radiate into the far-field, such as waveguide modes or surface plasmons, are radiative processes, since they contribute to $F$ and thus change the radiative decay rate $\Gamma_{\mathrm{r}}$. Furthermore, in practice the integration over $k_{\|}$is only carried out up to a finite cutoff value, where the contribution of surface plasmons has dropped to sufficiently small values (in most cases at around $\left.4 \cdot 10^{7} \mathrm{~m}^{-1}\right)$.

With the simulated power dissipation spectra, it is also possible to derive the fraction of power that is dissipated to the different optical modes as discussed at the beginning of this section:

$$
\tilde{P}_{\text {mode }}=q \cdot \int_{\lambda_{1}}^{\lambda_{2}} S(\lambda) \int_{u_{1}}^{u_{2}} P\left(k_{\|}, \lambda, z\right) d k_{\|} d \lambda .
$$

In this equation $u_{i}$ denotes the limits for the in-plane wavevector between which power is coupled to the different optical channels as is already indicated by the white lines in Fig. 4. For example, if the power of the direct emission $\left(\tilde{P}_{\text {air }}\right)$ is of interest (region 1 in Fig. 4), the in-plane wavevector has to fulfill the condition $0 \leq k_{\|} \leq n_{\text {air }} \cdot k_{0}$, with $n_{\text {air }}$ being the refractive index of air and $k_{0}$ is the wavevector in air for a given wavelength $\left(k_{0}=2 \pi / \lambda\right)$. Moreover, in the case of emission to air, this simulation yields directly the outcoupling factor $\eta_{\text {out }}$ for the device without light extraction modifications, if the radiative quantum efficiency of the emitting system is set to unity:

$$
\eta_{\text {out }}=\frac{\tilde{P}_{\text {air }}}{\tilde{P}_{\text {tot }}} ; \text { if } q \equiv 1 .
$$

Otherwise, if $\mathrm{q}<1$, one has to consider the effective RQE, too, leading to the following equation:

$$
q_{\text {eff }} \cdot \eta_{\text {out }}=\frac{\tilde{P}_{\text {air }}}{\tilde{P}_{\text {tot }}} ; \text { if } q \neq 1 .
$$

However, even in this case it is straightforward to obtain $\eta_{\text {out }}$ by using Eq. 8. Furthermore, it is also possible to calculate the outcoupling efficiency of the device, if macroscopic outcoupling enhancements such as index-matched glass hemispheres are used. If so, one has to change the upper limit of the integration over the in-plane wavevector from $n_{\text {air }} \cdot k_{0}$ 
to $n_{\text {substrate }} \cdot k_{0}$, with $n_{\text {substrate }}$ being the refractive index of the used substrate (region $1+2$ in Fig. 4). Please note that in an experiment $\eta_{\text {out }}$ can be lower than the calculated one, e.g. due to refractive index mismatch of the outcoupling structure. Another challenge for simulation tools exists for non-isotropic emitter orientations. In this case, one has to distinguish between three different fundamental orientations of transition dipole moments inside the OLED and perform the simulation for each of these three dipoles. The differently oriented dipoles are denoted as

- $P_{\perp, \mathrm{TM}}$ : dipoles oriented perpendicular to the substrate plane (vertical; $z$-direction), which emit p-polarized (transverse magnetic (TM)) light,

- $P_{\|, \mathrm{TM}}$ : dipoles oriented parallel to the substrate plane (horizontal; $x-y$ plane), which emit p-polarized (TM) light,

- $P_{\|, \mathrm{TE}}$ : dipoles oriented parallel to the substrate plane (horizontal; $x$ - $y$ plane), which emit s-polarized (transverse electric(TE)) light.

Due to symmetry aspects the two horizontally oriented dipole orientations are equivalent, because the plane of observation is chosen arbitrarily. Therefore, in the case of isotropic emitter orientation the total emitted power by an ensemble of excited molecules inside the OLED cavity can be written as:

$$
P_{\text {iso }}=\frac{2}{3} P_{\|}+\frac{1}{3} P_{\perp} .
$$

To account for orientation effects, it is helpful to introduce an anisotropy factor $\theta$ that is defined as the ratio between the amount of vertical dipoles and the total number of dipoles[36]. According to this definition $\theta$ becomes 1/3 for isotropic emitter orientation, 1 for only vertical dipoles (dipoles in $z$-direction) and 0 for totally horizontal emitter orientation (all dipoles are lying in the $x-y$-plane). With this information the weighted Purcell factor can be calculated as

$$
F(\theta)=\frac{1-\theta}{2}\left(F_{x}+F_{y}\right)+\theta \cdot F_{z} .
$$

By this means separate Purcell factors $F_{i}$ are introduced for each of the three orthogonal dipole orientations $(x, y$, and $z)$

$$
F_{i}=\int_{\lambda_{1}}^{\lambda_{2}} S(\lambda) \int_{0}^{\infty} P_{i}\left(k_{\|}, \lambda, z\right) d k_{\|} d \lambda .
$$

Finally, the relative change of the excited states lifetime with respect to the value $\tau_{0}$ measured in the absence of the cavity $\left(F_{i} \equiv 1\right)$ is obtained by taking into account the radiative quantum efficiency of the emitter material $q$ from Eq. 2

$$
\frac{\tau(\theta)}{\tau_{0}}=\frac{\Gamma_{0}}{\Gamma(\theta)}=\frac{1}{F(\theta) \cdot q+(1-q)} .
$$

\section{EFFICIENCY ANALYSIS OF OLEDS}

Using this background, a comprehensive efficiency analysis for arbitrary emitter orientation can be performed. The stateof-the-art OLED stack under investigation and the intrinsic emitter spectrum are shown in Fig. 5. A hole injection and transport layer (HTL) is deposited onto an indium tin oxide (ITO) coated glass substrate followed by an electron blocking (a)

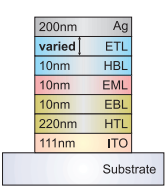

(b)

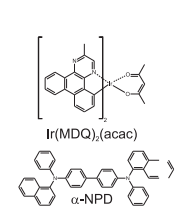

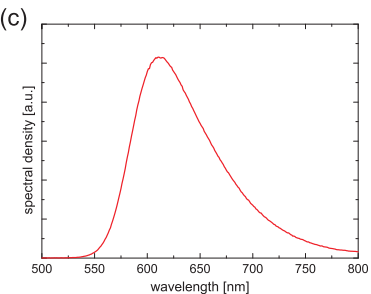

Fig. 5. (a) State-of-the-art device stack under investigation with varying electron transporting layer (ETL) thickness. The emission zone was assumed to be $\delta$-shaped in the middle of the emission layer (EML). (b) Molecular structure of the used emissive guest/host system containing $\operatorname{Ir}(\mathrm{MDQ})_{2}$ (acac) doped with $8 \mathrm{wt} \%$ into an $\alpha$-NPD matrix. (c) Intrinsic red emission spectrum of the emitting system. (see also Ref.[35]).

layer (EBL). Thereafter, an $\alpha$-NPD matrix doped with $8 \mathrm{wt} \%$ $\operatorname{Ir}(\mathrm{MDQ})_{2}$ (acac) (structure shown in Fig. 5) forms the thin emission layer (EML) of the device. In order to provide a high (close to unity) charge balance factor the emission layer is subsequently covered by an appropriate hole blocking layer (HBL) and an electron injection and transport layer (ETL) and finally contacted by a thick silver cathode. For more information about the device fabrication we refer to Ref. [35]. The essential method to analyze, e.g., the radiative quantum efficiency of an emissive guest/host system inside an OLED is a proper variation of their cavity length. Since the strength of the cavity depends on the distance between the emitter and the highly reflecting cathode of the device, the best results can be achieved by a variation of the ETL thickness[19], [28]. This in turn affects the power distribution between the different optical modes, the effective RQE and the excited states lifetime of the used guest-host system. Please note that due to conductivity doping of the transport layers electrical changes by layer thickness variation can be minimized. Thus, the charge balance factor and the singlet-triplet ratio in the equation for the external quantum efficiency (eq. 1) remain constant for all transport layer thicknesses and both are assumed to be one.

The prevailing emitter orientation can be figured out by means of three different experiments. The first method is a photoluminescence measurement of the angular dependent emission spectrum in the absence of the metallic cathode. In this case, the $p_{z}$ dipoles cannot couple to plasmons and will emit light under high emission angles[29]. The second method is an electroluminescence experiment. In this case it is mandatory to choose an appropriate ETL thickness[43], [44]. Due to different interference conditions for horizontally and vertically oriented dipoles, there are emitter to cathode distances where direct emission of the $p_{x^{-}}$and $p_{y}$-dipoles is almost completely suppressed, while direct emission of the $p_{z}$-dipoles is enhanced [31]. The third method consits of determining the optical anisotropy by ellipsometry[34]. However, this method is not practical for analyzing the emitter orientation in dye-doped guest/host systems.

\section{A. Changes of excited states lifetimes in OLEDs}

The different interference conditions for the three different emitter orientations can be seen in the relative excited states lifetime changes $\left(\tau / \tau_{0}\right)$ with the ETL thickness. Figure 6 


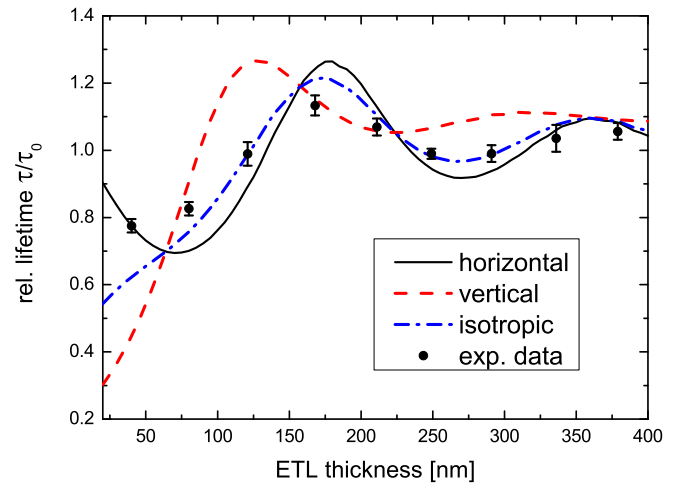

Fig. 6. Separately simulated relative lifetime changes as a function of the electron transport layer thickness for horizontal, vertical and isotropic emitter orientation. The intrinsic radiative quantum efficiency was set to unity $(\mathrm{q}=1)$ (see also Ref.[31]). The dots are measured photoluminescence lifetimes normalized by $\tau_{0}=1.37 \mu \mathrm{s}$.

shows this behavior for an assumed intrinsic radiative quantum efficiency of $q=1$. Also included in this plot are measured photoluminscence lifetimes normalized by $\tau_{0}=1.37 \mu \mathrm{s}$. From eq. 15 it follows, that these relative lifetime changes are the reciprocal values of the Purcell factor $F$. Due to the different interference conditions for vertical and horizontal dipoles, the minima of the Purcell factors are at different positions inside the cavity. While horizontal and isotropic oriented emitters show their minimum for $F$ at emitter/cathode distances of about $180 \mathrm{~nm}$, it is located at $125 \mathrm{~nm}$ for the vertically oriented dipoles. However, the behavior gets even more significant for small emitter-cathode distances. Whereas horizontal dipoles only couple very weakly to surface plasmons, the vertical dipoles are dissipating almost all their energy to plasmons resulting in a very high Purcell factor and so in a strong reduction of their intrinsic lifetime[26], [57]. This is due to the intrinsic radiation pattern of an electrical dipole, that emits most of its energy perpendicular to the dipole orientation.

The comparison between experimental data and simulation results demonstrates that none of the three limiting cases of emitter orientation with $q=1$ yields a good description. Thus, either the intrinsic radiative quantum efficiency of the used guest/host system is not unity and/or the prevailing emitter orientation is not isotropic.

Please note, that this simulation is only shown for emitter/cathode distances larger than $20 \mathrm{~nm}$. For smaller ETL thicknesses the used dipole model is not valid and an energy transfer to lossy surface waves in the metal will occur[21], [27], [58].

\section{B. Power distribution between optical modes in OLEDs}

The variation of the cavity length does not only change the radiative rate of the excited states and therewith the effective RQE of an emitting species inside an OLED stack, but also the power distribution between the different optical modes. To analyze this behavior in more detail, simulations of the power distribution between all optical modes for $\mathrm{q}=1$ and
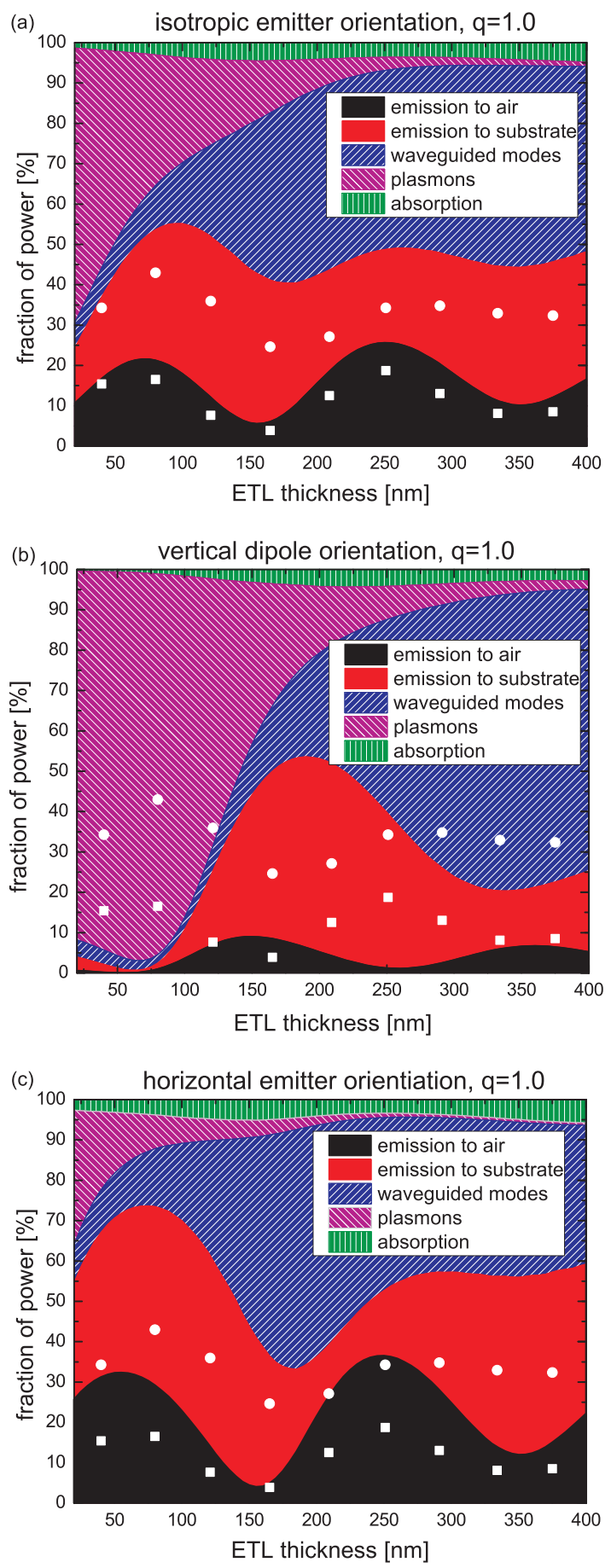

Fig. 7. Simulations of the power distribution between the different optical modes for an RQE of unity as a function of the ETL thickness, if a) an isotropic, b) a vertical and c) a horizontal emitter orientation is assumed(see also Ref.[31]). Measured EQE values with and without macroextractor for a current density of $1 \mathrm{~mA} / \mathrm{cm}^{2}$ are represented by white dots and squares, respectively.

for different emitter orientations (horizontal, isotropic and vertical) have been performed. The RQE was set to unity to simplify the discussion. However, the basic results are valid as long as an RQE over $\approx 50 \%$ is used in the actual device[25], which will be discussed in the following subsection. Figure 7 shows the results of these simulations together with measured $\mathrm{EQE}$ values with and without macroextractor for a low current 
density of $1 \mathrm{~mA} / \mathrm{cm}^{2}$ represented by white dots and squares, respectively. Note that in order to investigate the intrinsic RQE of the emitting system, as low current densities as possible should be used for the EQE analysis to avoid current induced quenching effects, such as triplet-polaron-quenching or triplettriplet-annihilation[23], [56], [41]. As a rule of thumb, the current should be chosen low enough to exclude an efficiency roll-off in the device under investigation and high enough to minimize uncertainties of the measuring procedure. Hence, a value of $1 \mathrm{~mA} / \mathrm{cm}^{2}$ turned out to be a reasonable compromise, however, this value can change for different OLED stacks.

Again, a clear difference between horizontally and vertically oriented dipoles can be identified for all simulated ETL thicknesses. While the maxima in the EQE with and without substrate modes are at almost the same ETL thicknesses in the isotropic and the horizontal case, the maxima for vertical dipole orientation are at completely different positions. For vertically oriented dipoles the direct emission cannot reach the $10 \%$ mark and is the highest for an ETL thickness of $150 \mathrm{~nm}$, where horizontally oriented dipoles have their minimum. Thus, experiments for determining the prevailing emitter orientation should be performed at that particular ETL thickness[43], [44]. If the substrate modes can be coupled out with e.g. a macroextractor, a suprisingly high $\mathrm{EQE}$ value of $53 \%$ can be reached for the vertical case at about $180 \mathrm{~nm}$ ETL thickness. Nevertheless, the vertically oriented dipoles are not favourable at all for OLED applications in displays or for lighting due to the strong coupling to surface plasmons especially for ETL thicknesses smaller than $100 \mathrm{~nm}$ and their strong angular dependent emission spectra. In the isotropic case, higher results for the EQE at different ETL thicknesses can be reached for both cases. Hence, $26 \%$ and $55 \%$ of the generated light can be coupled out for the direct emission and if a macroextractor is used, respectively. However, the best results for the EQE of an OLED can be achieved for completely horizontal dipole orientation, where $30 \%$ are achieved for direct emission and the application of a macroextractor can remarkably yield $74 \%$ external quantum efficiency[31]. Please note that the optimal ETL thickness in the isotropic and the horizontal case depends on the intended light outcoupling method. If only direct emission is of interest, the second cavity maximum yields the best result. If outcoupling enhancements are used, and therewith the substrate modes are extracted to air, the first cavity maximum is the favourable one.

However, as in the case of the excited states lifetimes, the simulations do not describe the measured EQE values in a proper way (see Fig. 7). Therefore, one has to conclude that the intrinsic radiative quantum efficiency of the used guest/host system is not unity and/or the prevailing emitter orientation is not isotropic as will be discussed in more detail below.

\section{Influence of the intrinsic radiative quantum efficnecy}

Figure 8 shows the direct emission of the OLED stack under investigation with an isotropic emitter orientation as a function of the ETL thickness and the RQE of the emitting system. It can clearly be seen that the second cavity maximum of the emission is only higher than the first maximum for values

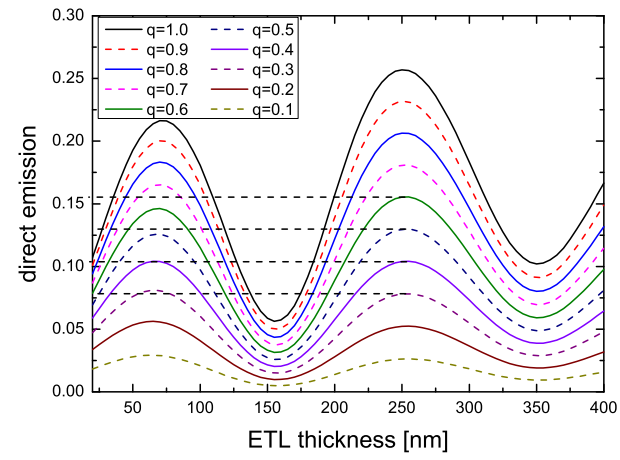

Fig. 8. Simulated direct emission of the OLED stack under investigation for an isotropic emitter orientation as a function of the ETL thickness and different RQEs.

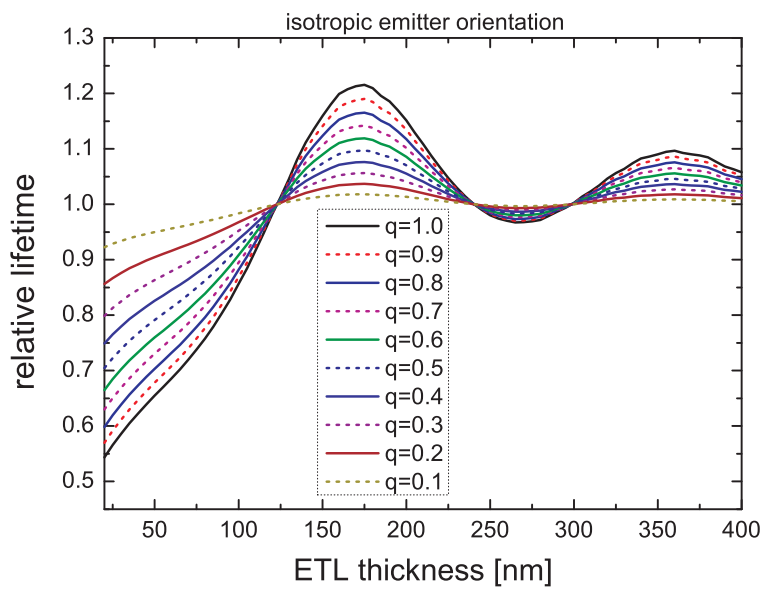

Fig. 9. Simulated relative changes of the excited states lifetime of the emitting system as a function of the ETL thickness and for different radiative quantum efficiencies $q$ and an isotropic emitter orientation.

of the RQE higher than $40 \%$. If the RQE is less than $40 \%$ the first maximum would be the favourable one, and so the ETL thicknesses for a maximum EQE are not only depending on the emitter orientation, but also on the intrinsic radiative quantum efficiency of the emitting system. Please note that this effect should be taken into account for OLED optimization by means of layer thickness variation for the relevant operation conditions, because a reduction of the RQE due to TTA or TPQ especially at high current densities and therewith high brightness can shift the optimal emitter position inside the OLED from the second to the first cavity maximum[28].

Figure 9 demonstrates the behavior of the relative changes of the excited states lifetime as a function of the emitter/cathode distance for different RQEs and an isotropic emitter orientation. It can clearly be seen, that the oscillations of the excited states lifetime are damped for decreasing radiative quantum efficiencies, which is obvious from Eq. 15. Hence, fitting the measured excited states lifetimes at varying ETL thicknesses with $q$ and $\tau_{0}$ as the only free parameters results in the apparent $\mathrm{RQE}$ of the emitting system. Please note that the measured data points should be located especially at small ETL thicknesses and in the 


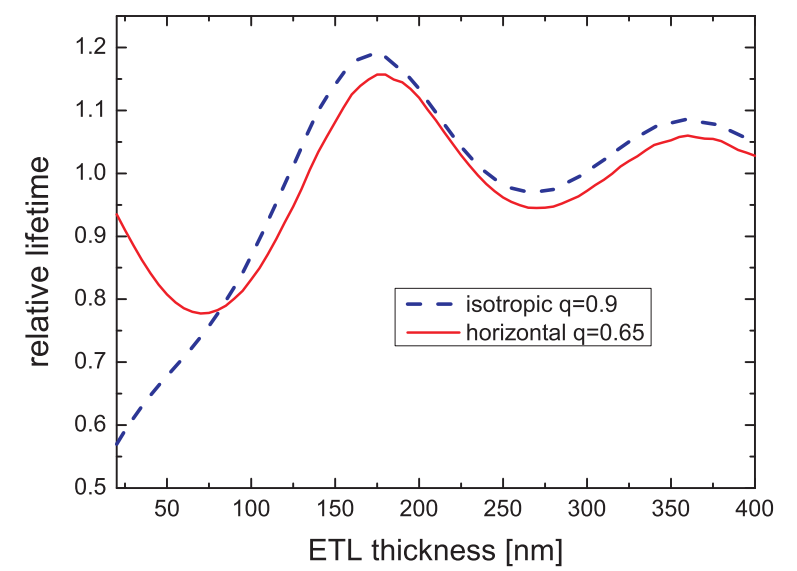

Fig. 10. Simulation of the relative lifetime changes for the OLED stack under investigation as a function of the ETL thickness and for different emitter orientations with different RQEs (see also Ref.[31]).

maxima of the relative excited states lifetime changes because at high emitter/cathode distances the differences between the simulated RQEs are vanishing.

\section{Influence of non-isotropic emitter orientation}

As pointed out in the last subsections, it is a crucial point to determine the RQE and the prevailing emissive dipole orientation of an emitting system inside a complex OLED structure to achieve a consistent efficiency analysis. Figure 10 demonstrates a possible ambiguity for the determination of the RQE of the emitting system, if the emissive dipole orientation is not known. Here, one can not clearly distinguish between an isotropic emitter orientation with an RQE of $90 \%$ and a completly horizontal emitter orienation with a $q$ of $65 \%$ for ETL thicknesses larger than $100 \mathrm{~nm}$, resulting in a huge difference for the derived value of $q$. Therefore, it is mandatory to measure the relative changes of the excited states lifetime especially for small emitter/cathode distances $(\leq 80 \mathrm{~nm})$ because in this regime this measurement is very sensitive for orientation effects as discussed in subsection A.

The situation becomes even more significant, if the EQE with and without an outcoupling structure for different ETL thicknesses are measured. Figure 11 demonstrates the behavior of the EQE as a function of the emitter/cathode distance for the same assumptions about the emissive dipole orientation and identical RQEs as in Fig. 10. While the simulation yields very similar results for the direct emission for ETL thicknesses larger than $75 \mathrm{~nm}$ for both orientations with the corresponding RQEs, the behavior for the outcoupled substrate modes is significantly different. This emphasizes again the importance of performing all possible measurements to achieve a consistent efficiency analysis of OLEDs. E.g. if one is not including orientation effects for external quantum efficiency measurements of the direct emission, a huge overestimation of the corresponding RQE would follow. Therefore, it is a cruial point to analyze the substrate modes of the device, because EQE measurements are very sensitive for emitter orientation if outcoupling structures are used. However, it is remarkable

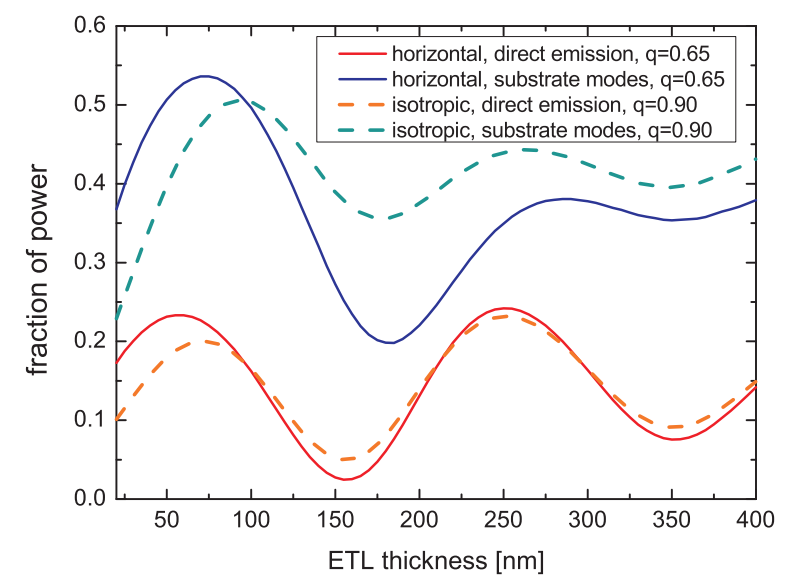

Fig. 11. Simulation of the EQE with and without an outcoupling structure for the OLED stack under investigation as a function of the ETL thickness and for different emitter orientations with different RQEs (see also Ref.[31]).

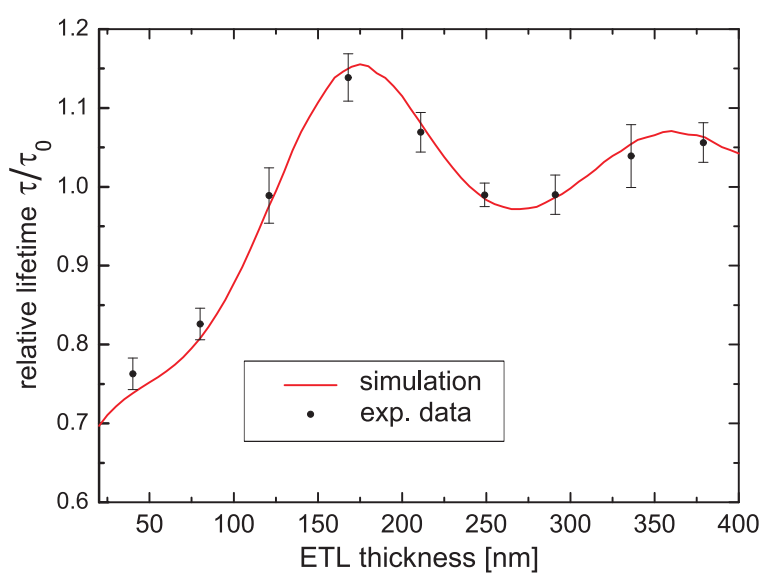

Fig. 12. Dots: Measured phosphorescence lifetimes normalized to the intrinsic value $\tau_{0}=1.37 \mu \mathrm{s}$. Line: Best fit for a predominantly horizontal orientation with $\theta=0.24$ yielding $q=0.7$ (see also Ref.[36]).

that a horizontal emitter orientation can achieve the same or even higher values of the EQE although the assumed RQE of the emitting system is much lower than in the isotropic case.

\section{E. Comprehenisve efficency analysis including emitter orien- tation}

In order to verify this simulation based approach, the OLED stack shown in Fig. 5 has been investigated with nine different ETL thicknesses. Independently, the emitter orientation of the emitting system was determined by radiation pattern analysis yielding an emitter orientation of 0.63 vertically to 2 horizontally oriented dipoles, resulting in an anisotropy factor of $\theta=0.24$ [35]. With this information it is now possible to determine the RQE of the emissive species inside the OLED cavity via the two presented methods.

Figure 12 shows the results of the measured photoluminescence lifetime of the excited molecules for the different ETL thicknesses normalized with the intrinsic value $\left(\tau_{0}=1.37 \mu \mathrm{s}\right)$. The best fit for the prevailing, predominantly horizontal emitter orientation with $\theta=0.24$ yields an RQE value of $70 \%$. The simulated behavior of the excited states lifetimes can describe the measured data points very well, especially for 


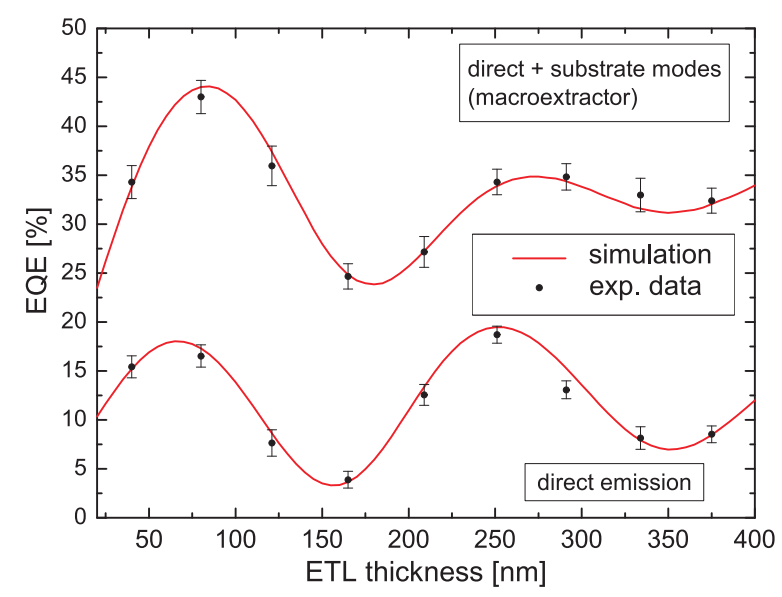

Fig. 13. Dots: Measurements of the external quantum efficiency for a current density of $1 \mathrm{~mA} / \mathrm{cm}^{2}$ for different ETL thicknesses with (top) and without (bottom) outcoupling enhancement. Line: Simulated behavior for the stack under investigation as a function of the ETL thickness for an predominantly horizontal $(\theta=0.24)$ emitter orientation with $q=0.7$ (see also Ref.[36]).

low emitter/cathode distances, where this method is very sensitive for emitter orientation. This confirms the determined predominantly horizontal emitter orientation for this emitting system.

The results of the EQE measurements are shown in Figure 13. The measurements have been performed in an integrating sphere for low current density of $j=1 \mathrm{~mA} / \mathrm{cm}^{2}$ to avoid quenching effects due to TPQ and TTA. Both data sets, the emission without and with macro-extractor, can be described consistently with a predominantly horizontal emitter orientation $(\theta=0.24)$ and an RQE of $70 \%$. This is in perfect agreement with the results from the excited states lifetime.

Thus, both measurements lead to a lower intrinsic radiative quantum efficiency of the used guest/host system than previously reported values by other groups, who have assumed an isotropic emitter orientation[59], [46]. This clearly demonstrates the importance of taking into account possible deviations from randomness in the emitter orientation to ensure a consistent efficiency analysis in state-of-the-art organic lightemitting diodes based on small molecules.

\section{DEgRADATION ANALYSIS OF OLEDS}

Another limiting factor for commercial applications of OLED technology in the general lighting sector is their comparatively short lifetime at high brightness. Although first white OLEDs, achieving several thousand hours for LT70 (LT70 is defined as the time after that a luminance drop by $30 \%$ for a constant current density is reached), have been established in the market by several companies, this value is still not satisfactorily. Thus, analyzing and understanding degradation effects during electrical operation is of paramount importance for further progress in the field.

Therefore, we have introduced a new approach to quantify the changes of the factors determining the EQE during electrical operation[60]. Hence, we have performed the same kind of efficiency analysis as described in section IV in the pristine state and after an accelerated degradation process at high

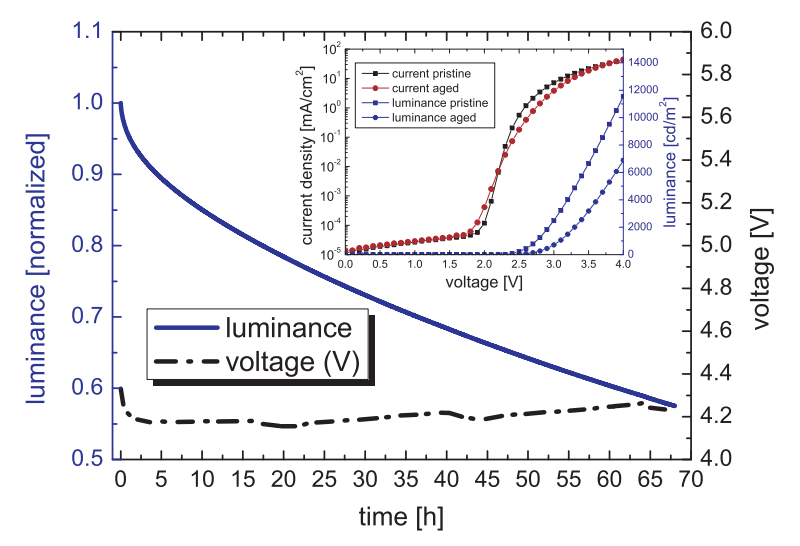

Fig. 14. Exemplary degradation behavior for a constant current density of $j=62.5 \mathrm{~mA} / \mathrm{cm}^{2}$ of the OLED under investigation containing an ETL thickness of $249 \mathrm{~nm}$. Blue solid line: Decrease of the normalized luminance with time reaching LT60 after 65 hours operation time. Black, dashed line: Increase of the required voltage during degradation. The inset shows the IVLcharacteristics for the pristine state and after degradation(see also Ref.[60]).

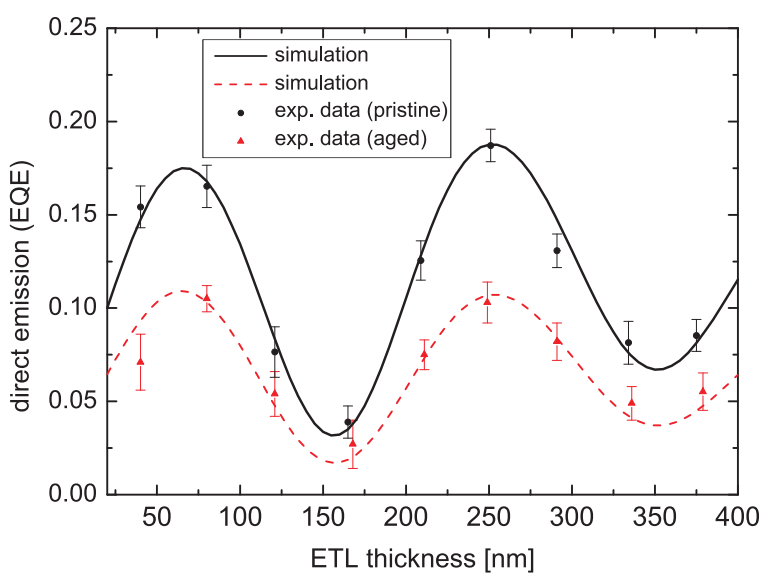

Fig. 15. Determination of radiative quantum efficiency for the OLED under investigation via external quantum efficiency measurements without outcoupling structures (direct emission). Black dots represent the measurements for the pristine devices, red triangles for the aged OLEDs. Black solid line and red dashed line are simulations for different quantum efficiencies of 0.68 and 0.4 , respectively. The simulations have been performed for an emitter orientation of $\theta=0.24$ (see also Ref.[60]).

current densities.

Figure 14 shows an exemplary degradation curve of the OLED shown in Fig. 5 with an ETL thickness of about $250 \mathrm{~nm}$. The degradation was performed by applying a constant current density of $j=62.5 \mathrm{~mA} / \mathrm{cm}^{2}$ to the OLED resulting in an initial luminance of about $15.000 \mathrm{~cd} / \mathrm{m}^{2}$. After an electrical stressing time of about $65 \mathrm{~h}$ the luminance dropped to $60 \%$ of the initial value (LT60). Although this huge decrease of the luminance is observed, the required voltage remains almost constant over the whole degradation process and is only slightly increased. Thus, the changes in the current-voltagecharacteristics (IV) are less pronounced than in the luminance.

Interestingly, the relative luminance drop for the devices containing different ETL thicknesses after degradation under the same current flow is identical, even though the different positions of the emission layer inside the OLED cavity are drastically changing the outcoupling factor and the effective RQE of the devices. Thus, an efficiency analysis as presented 


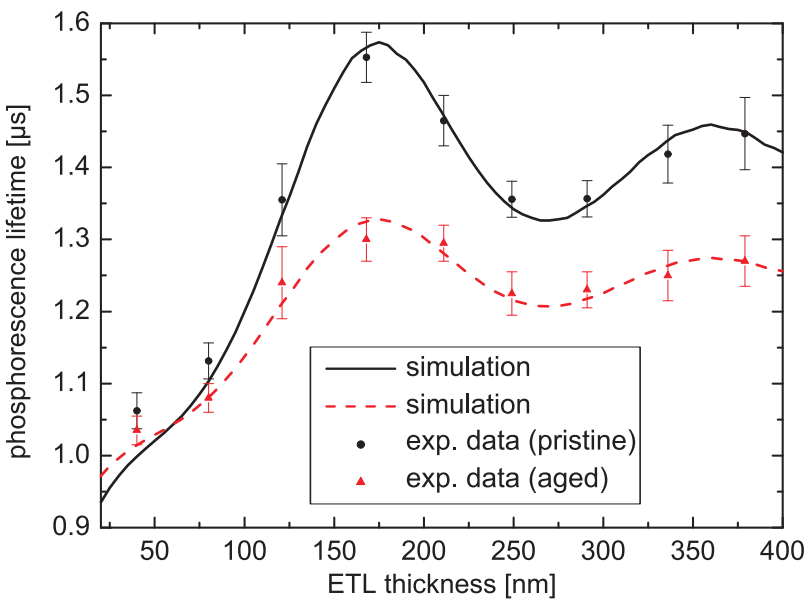

Fig. 16. Changes of the photoluminescence excited states lifetime as a function of the ETL thickness. Black dots represent pristine devices, red triangles aged OLEDs. The lines are fits for the pristine state (black, solid) and after degradation (red, dashed) resulting in different RQEs and intrinsic lifetimes(see also Ref.[60]).

in the previous section is possible after degradation. Figure 15 shows the compilation of the external quantum efficiencies before and after degradation for the direct emission of the OLEDs measured at a low current density of $1 \mathrm{~mA} / \mathrm{cm}^{2}$ to avoid quenching effects. The comparison of the EQE measurements with the simulations yields an RQE of $(68 \pm 2) \%$ for the pristine state and $(40 \pm 2) \%$ after degradation, where in both cases the same emitter orientation is included. Thus, the whole luminance drop can be explained by a decrease in the RQE of the emitting system, while other factors determining the EQE seem to remain constant and are not influenced in a measureable way. To ensure a reliable determination of the RQE, the emitter orientation was independently determined by radiation pattern analysis in the cavity minimum for an ETL thickness of $160 \mathrm{~nm}$. However, no changes of the emitter orientation are detectable for the aged device.

Finally, the determination of the RQE via the excited states lifetime for the pristine state and after degradation has been carried out. Please note that the time-resolved measurements have been performed by laser excitation and not electrically pumped. As mentioned before in this article, the excitation by electrical pulses would yield better results, however, due to the constant emission zone over the thin emission layer $(10 \mathrm{~nm})$ laser excitation is an acceptable method. The fitting procedure of the measured values with simulated data results in an RQE of $(70 \pm 5) \%$ with an intrinsic lifetime of $(1.37 \pm 0.03) \mu$ s for the pristine devices and a $q$ of $(40 \pm 5) \%$ with a modified intrinsic lifetime of $(1.23 \pm 0.03) \mu$ s after degradation. These results are in excellent agreement with the values from EQE measurements. Moreover, the changed intrinsic lifetime after degradation gives a hint for modified radiative and nonradiative decay rates of the emitting species. However, this result is beyond the scope of this article and for more details we refer to Ref.[60].

\section{CONCLUSiON}

In conclusion, we have shown that optical simulations are an indispensable tool for analyzing the emission properties of organic light-emitting diodes. We have figured out the importance of emitter orientation and how it is possible to implement this feature to established simulation tools. Furthermore, we have presented a method for a determination of the intrinsic radiative quantum efficiency (RQE) of emitting systems. Therewith it is possible to perform a comprehensive efficiency analysis based on a systematic variation of the emitter/cathode distance to distinguish between cavity effects, which are influencing the effective RQE of an emitting system and power distributions between different optical modes, that are changing the outcoupling factor of the device. Finally, we have discussed the application of the efficiency analysis to degradation experiments.

\section{ACKNOWLEDGMENT}

The authors would like to acknowledge financial support by the German Federal Ministry of Education and Research (BMBF) under the Contract No. FKZ 13N10474 (TOPAS 2012). Christian Mayr acknowledges financial support by the Bayerische Forschungsstiftung. We are grateful to colleagues from Osram Opto Semiconductors (Regensburg, Germany) and Fraunhofer Institute for Applied Optics and Precision Engineering (Jena, Germany) for their fruitful discussions and continuous support to this work.

\section{REFERENCES}

[1] M. Pope, H.P. Kallmann, and P. Magnante, J. Chem. Phys. 38, $2042-$ 2043 (1963).

[2] C.W. Tang, and S.A. VanSlyke, Appl. Phys. Lett. 51, 913-915 (1987).

[3] J.H. Burroughes, D.D.C. Bradley, A.R. Brown, R.N. Marks, K. Mackay, R.H. Friend, P.L. Burns, and A.B. Holmes, Nature 347, 539 (1990).

[4] http://www.oled-info.com/osram-developed-87-lmw-white-oled-panelproduction-soon

[5] R. Cheng, Wall Street Journal, September 6th (2010).

[6] Y.-S. Tyan, J. Photon. Energy 1, 011009 (2011).

[7] Commercial white OLEDs are offered since 2010, e.g. by Osram and Philips (see www.osram.com and www.lighting.philips.com) .

[8] T. Tsutsui, E. Aminaka, C.P. Lin, and D.-U. Kim, Trans. R. Soc. Lond. A 355, 801-814 (1997).

[9] M. Pfeiffer, S.R. Forrest, K. Leo, and M.E. Thompson, Adv. Mater. 14, 1633-1636 (2002).

[10] C. Adachi, M.A. Baldo, M.E. Thompson, and S.R. Forrest, J. Appl. Phys. 90, 5048 (2001).

[11] M.A. Baldo, D.F. O'Brien, M.E. Thompson, and S.R. Forrest, Phys. Rev. B 60, 14422-14428 (1999).

[12] M. Segal, M.A. Baldo, R.J. Holmes, S.R. Forrest, and Z.G. Soos, Phys. Rev. B 68, 075211-14 (2003).

[13] D.Y. Kondakov, T.D. Pawlik, T.K. Hatwar, and J.P. Spindler, J. Appl. Phys. 106, 124510-7 (2009).

[14] Y. Luo, and H. Aziz, Adv. Funct. Mater. 20, 1285-1293 (2010).

[15] D. Yokoyama, Y. Park, B. Kim, S. Kim, Y.-J. Pu, and J. Kido, Appl. Phys. Lett. 99, 123303 (2011).

[16] Y.-J. Pu, G. Nakata, F. Satoh, H. Sasabe, D. Yokoyama, and J. Kido, Adv. Mater. 24, 1765-1779 (2012).

[17] A. Endo, K. Sato, K. Yoshimura, T. Kai, A. Kawada, H. Miyazaki, and C. Adachi, Appl. Phys. Lett. 98, 083302 (2011).

[18] K. Goushi, K. Yoshida, K. Sato, and C. Adachi, Nature Photon. 6, 253258 (2012).

[19] B.C. Krummacher, S. Nowy, J. Frischeisen, M. Klein, and W. Brütting, Org. Electron. 10, 478-485 (2009).

[20] S. Nowy, B.C. Krummacher, J. Frischeisen, N.A. Reinke, and W. Brütting, J. Appl. Phys. 104, 123109-9 (2008). 
[21] H. Becker, S.E. Burns, and R.H. Friend, Phys. Rev. B 56, 1893-1905 (1997).

[22] J.A.E. Wasey, A. Safonov, I.D.W. Samuel, and W.L. Barnes, Phys. Rev. B 64, 205201-9 (2001).

[23] N.C. Giebink, and S.R. Forrest, Phys. Rev. B 77, 235215 (2008)

[24] N.C. Greenham, R.H. Friend, and D.D.C. Bradley, Adv. Mater. 6, 491494 (1994).

[25] S. Nowy, J. Frischeisen, and W. Brütting, Proc. SPIE 7415, 74151C (2009).

[26] L.H. Smith, J.A.E. Wasey, I.D.W. Samuel, and W.L. Barnes, Adv. Funct. Mater. 15, 1839-1844 (2005)

[27] W.L. Barnes, J. Mod. Opt. 45, 661-699 (1998).

[28] D.S. Setz, T.D. Schmidt, M. Flämmich, S. Nowy, J. Frischeisen, B.C. Krummacher, T. Dobbertin, K. Heuser, D. Michaelis, N. Danz, W. Brütting, and A. Winnacker, J. Photon. Energy 1, 011006 (2011).

[29] J. Frischeisen, D. Yokoyama, C. Adachi, and W. Brütting, Appl. Phys. Lett. 96, 073302 (2010)

[30] J. Frischeisen, D. Yokoyama, A. Endo, C. Adachi, and W. Brütting, Org. Electron. 12, 809.-17 (2011).

[31] T.D. Schmidt, M. Flämmich, B.J. Scholz, D. Michaelis, C. Mayr, N. Danz, and W. Brütting, Proc. SPIE 8435, 843513 (2012).

[32] J.A.E. Wasey, A. Safonov, I.D.W. Samuel, and W.L. Barnes, Opt. Commun. 183, 109-121 (2000).

[33] M. Flämmich, M.C. Gather, N. Danz, D. Michaelis, A.H. Bräuer, K. Meerholz, and A. Tünnermann, Org. Electron. 11, 1039-1046 (2010).

[34] D. Yokoyama, J. Mater. Chem. 21, 19187 (2011).

[35] M. Flämmich, J. Frischeisen, D.S. Setz, D. Michaelis, B.C. Krummacher, T.D. Schmidt, W. Brütting and N. Danz, Org. Electron. 12, 1663-1668 (2011).

[36] T.D. Schmidt, D.S. Setz, M. Flämmich, J. Frischeisen, D. Michaelis, B.C. Krummacher, N. Danz, and W. Brütting, Appl. Phys. Lett. 99, 163302 (2011).

[37] K.A. Neyts, J. Opt. Soc. Am. A 15, 962-971 (1998).

[38] B. Ruhstaller, T. Beierlein, H. Riel, S. Karg, C.J. Scott, and W. Riess, Selec. Top. Quan. Electron. of IEEE 9, 723-731 (2003).

[39] C.-C. Wu, C.-L. Lin, P.-Y. Hsieh, and H.-H. Chiang, Appl. Phys. Lett. 84, 3966 (2004).

[40] C.-C. Lee, M.-Y. Chang, P.-T. Huang, Y.C. Cheng, Y. Chang, and S.W. Liu, J. Appl. Phys. 101, 114501 (2007).

[41] S. Reineke, K. Walzer, and K. Leo, Phys. Rev. B 103, 044509 (2008).

[42] S.L.M. van Meensfort, M. Carvelli, M. Megens, D. Wehenkel, M. Bartyzel, H. Greiner, R.A.J. Janssen, and R. Coehoorn, Nature Photon. 4, 329-335 (2010).

[43] M. Flämmich, D. Michaelis, and N. Danz, Org. Electron. 12, 83-91 (2011).

[44] M. Flämmich, D. Michaelis, and N. Danz, Proc. SPIE 7954, 795410 (2011).

[45] E. Nichelatti, F. Bonfigli, M.A. Vincenti, and R.M. Montereali, J. Opt. Tech. 78, 424-429 (2011).

[46] M. Furno, R. Meerheim, S. Hofmann, B. Lüssem, and K. Leo, Phys. Rev. B 85, 115205 (2012).

[47] W. Brütting, J. Frischeisen, T.D. Schmidt, B.J. Scholz, and C. Mayr, Phys. Status Solidi A 210, 44-65 (2013).

[48] E.M. Purcell, Phys. Rev. 69, 681 (1946).

[49] K.H. Drexhage, J. Lumin. 1-2, 693-701 (1970).

[50] K.H. Drexhage, Prog. Opt. 12, 165 (1974).

[51] R.R. Chance, A. Prock, and R. Silbey, J. Chem. Phys. 60, 2744-2748 (1974).

[52] R.R. Chance, A. Prock, and R. Silbey, Adv. Chem. Phys. 37, 1-65 (1978).

[53] W. Lukosz, J. Opt. Soc. Am. 71, 744-754 (1981).

[54] J.A.E. Wasey, and W.L. Barnes, J. Mod. Opt. 47, 725-741 (2000).

[55] M.C. Gather, M. Flämmich, N. Danz, D. Michaelis, and K. Meerholz, Appl. Phys. Lett. 94, 263301 (2009).

[56] N.C. Giebink, B.W.D. Andrada, M.S. Weaver, P.B. Mackenzie, J.J. Brown, M.E. Thomson, and S.R. Forrest, J. Appl. Phys. 103, 044509 (2008).

[57] J.-S. Kim, P.K.H. Ho, N.C. Greenham, and R.H. Friend, J. Appl. Phys. 88, 1073-1081 (2000).

[58] K. Celebi, D.T. Heidel, and M.A. Baldo, Opt. Express 15, 1762-772 (2007).

[59] R. Meerheim, M. Furno, S. Hofmann, B. Lüssem, and K. Leo, Appl. Phys. Lett. 97, 253305 (2010).

[60] T.D. Schmidt, D.S. Setz, M. Flämmich, B.J. Scholz, A. Jaeger, C. Diez, D. Michaelis, N. Danz, and W. Brütting, Appl. Phys. Lett. 101, 103301 (2012).

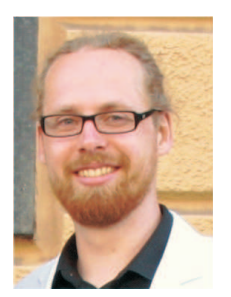

Tobias D. Schmidt is a $\mathrm{PhD}$ student at the University of Augsburg, Germany. He graduated from teaching physics and mathematics in 2008 . His main field of research covers organic light-emitting diodes with a focus on efficiency analysis, excitonic lifetime changes, molecular orientation and degradation effects.

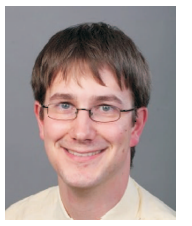

Bert J. Scholz is a PhD student in physics at the University of Augsburg, Germany. His research interest are optical simulations and the improvement of the light outcoupling efficiency of OLEDs, the role of waveguide modes and surface plasmon polaritons.

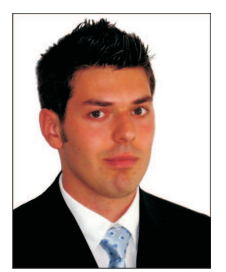

Christian Mayr is a PhD student at the University of Augsburg, Germany. He graduated in physics in 2011. His research interest is the understanding and control of molecular orientation in thin films for application in organic light-emitting diodes.

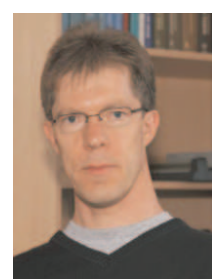

Wolfgang Brütting received his $\mathrm{PhD}$ in Physics from the University of Bayreuth in 1995 with a work on charge-density wave systems. Thereafter he moved to the field of organic semiconductors where he could take part in the development of organic light-emitting devices for display applications, inter alia as visiting scientist at Kyushu University (Japan) and IBM Zurich Research Laboratory (Switzerland). In 2003 he became Professor for Experimental Physics at the University of Augsburg. His current research activities include thin film growth, photophysics and electrical transport in organic semiconductor devices. 